\title{
General Psychiatry COVID-19 mental health care toolkit: an international collaborative effort by Early Career Psychiatrists section
}

\begin{abstract}
Frances Adiukwu (D , ${ }^{1}$ Laura Orsolini, ${ }^{2}$ Drita Gashi Bytyçi, ${ }^{3}$ Samer El Hayek, ${ }^{4}$ Jairo M Gonzalez-Diaz, ${ }^{5}$ Amine Larnaout, ${ }^{6}$ Paolo Grandinetti, ${ }^{7}$ Marwa Nofal, ${ }^{8}$ Victor Pereira-Sanchez, ${ }^{9}$ Mariana Pinto da Costa (D) , ${ }^{10,11,12}$ Ramdas Ransing, ${ }^{13}$ Andre Luiz Schuh Teixeira, ${ }^{14}$ Mohammadreza Shalbafan, ${ }^{15}$ Joan Soler-Vidal, ${ }^{16}$ Zulvia Syarif, ${ }^{17}$ Ganesh Kudva Kundadak, ${ }^{18}$ Rodrigo Ramalho ${ }^{19}$
\end{abstract}

To cite: Adiukwu F, Orsolini L, Gashi Bytyçi D, et al. COVID-19 mental health care toolkit: an international collaborative effort by Early Career Psychiatrists section. General Psychiatry 2020;33:e100270. doi:10.1136/ gpsych-2020-100270

Received 12 May 2020 Revised 15 July 2020 Accepted 17 August 2020
Check for updates

(c) Author(s) (or their employer(s)) 2020. Re-use permitted under CC BY-NC. No commercial re-use. See rights and permissions. Published by BMJ.

For numbered affiliations see end of article.

Correspondence to Dr Frances Adiukwu francesadiukwu@gmail.com

\section{ABSTRACT}

The collaborative effort of an international research team from the Early Career Psychiatrists section of the World Psychiatry Association has brought about an easy-to-use, quick and stepwise mental health care toolkit for the identification and appropriate referral of those in need of mental health care during the pandemic. This simple guide can be applied in the general outpatient setting and is catered for all healthcare professionals, regardless of their expertise within the mental health field with minimal training It is our hope that by incorporating this toolkit into our daily clinical care during the pandemic for high-risk patients and patients with non-specific complaints, we will be able to bridge the mental health gap present in our society.

\section{INTRODUCTION}

Following recent infectious disease outbreaks both at national (epidemic) and global levels (pandemic), there has been an increased preparedness by most countries against future outbreaks with a focus mainly on rapid case isolation, quarantine, vaccine, antiviral treatment and economic preparedness. However, little attention has been given to the acute and long-term mental health impact of such events. $^{12}$

In the current COVID-19 pandemic, there had been minimal mental health planning in advance of the pandemic, nor following the identification of the first cases in several countries such as India, Lebanon, as well as Nigeria. Despite research done following the Ebola outbreak in Nigeria in 2014 that pointed out an increased prevalence of psychological distress in survivors and relative(s) of those infected by the Ebola Virus, mental health planning and preparedness was not included in the COVID-19 preparedness plan. ${ }^{34}$ As a result, COVID-19 found most countries unprepared for the increased demand in mental health care brought by the pandemic.
Further to the development of a guiding framework for the implementation and evaluation of mental health care needs during the COVID-19 pandemic, ${ }^{5}$ we set out to prepare a stepped care toolkit by which the mental health challenges of different countries could be planned ahead irrespective of what phase of the pandemic they were in. Preventing and planning an integrated and multi-step approach through which the specific mental health challenges for each country, including the management of alcohol, substance use and behavioural addiction, could be properly addressed is fundamental as well as the development of alternative therapeutic and rehabilitative tools and a wider application and development of telepsychiatric means, particularly in those countries with already established service. ${ }^{6}$ Addressing COVID-19related stigma in such a framework is crucial, as stigma towards the infection, spread via misinformation and myths in mainstream and social media outlets, was also identified as a major factor implicated in the extent of the spread of the pandemic. This goes in concordance with lessons learnt from past infectious disease outbreaks. ${ }^{7}$ All the aforementioned primary mental health targets during the pandemic were the products of the collaborative effort of the Early Career PsychiatristWorld Psychiatric Association (ECP-WPA) research group. The research team held meetings via video conferencing and deliberated on the numerous gaps in mental health care during the pandemic from their country's perspectives and proposed strategies and interventions to bridge these gaps.

The collaborative effort of this research team has also brought about an easy-to-use, quick and stepwise mental health care toolkit for the identification and appropriate 
referral of those in need of mental health care during the pandemic (box 1). This simple guide can be applied in the general outpatient setting and is catered for all healthcare professionals, regardless of their expertise within the mental health field. It is our hope that by incorporating this guide into our daily clinical care during the pandemic, we will be able to bridge the mental health gap present in our society.

\section{Rationale for stepped care mental health toolkit}

During pandemics, mental health services are often inadequate and insufficient. Redeployment of mental health professionals to frontline healthcare workers also adds to the decreased mental health human resources that are available. $^{8}$ Low-income and middle-income countries (LMICs) with already inadequate mental health services are faced with the increase in prevalence of psychosocial distress in the general population and the worsening of the mental health gap already present. ${ }^{9-11}$ With insufficient number of qualified mental health providers and significant disparity in mental health services between urban and rural settings, there will be an increased dependence on primary healthcare as well as telemedicine services for accessing mental healthcare. ${ }^{12}$ There is, thus the need of a stepped care approach using a mental health toolkit that highlights the most prevalent psychosocial problems during the current COVID-19 pandemic (from reviewed literature) which could aid in bridging the mental health gap in LMIC as well as improve mental health service delivery in high-income countries by providing mental health services at the primary and community healthcare levels with appropriate referral to specialised mental health facilities as needed.

\section{Training of healthcare workers}

In order to achieve reliable screening results, healthcare workers at the primary healthcare and community healthcare levels would require some form of training on the use of the psychometric tools by mental health professionals (psychiatrists, clinical psychologist or psychiatric nurses) using face-to-face or telecommunications means (to maintain physical distancing required during this pandemic). It has been shown that with as little as 3 hours of training on the use of psychometric tools, inter-rater reliability of up to $81 \%$ can be achieved. ${ }^{13}$ These trainings can be reinforced periodically using pre-recorded training videos. All psychometric tools used in this toolkit have been validated in different countries and in different settings including primary care settings.

\section{Sampling of at-risk groups}

During infectious disease outbreaks and pandemics, the at-risk populations (group of individuals most susceptible to the infection during the outbreak ${ }^{14}$ ) are at increased risk of the infectious disease and also of the psychosocial consequences associated with the infectious disease. The at-risk populations often become hard to reach due to stigma, and physical and social distancing (which could be

\section{Box 1 COVID-19 mental health care toolkit}

\section{STEP I}

Identification of at-risk groups for mental health disorders during the different phases of the pandemic, bearing in mind the emotional epidemic curve as proposed by the ECP research group. ${ }^{5}$ Sensitisation and recruitment of patients for screening can be carried out using the previously suggested methods. In settings where a database of service users already exists, this can be used to identify and screen at-risk populations.

1. Persons under quarantine.

2. Family members/relatives/close contacts potentially at high risk for COVID-19 infection.

3. Persons who have tested positive for COVID-19 (asymptomatic and symptomatic).

4. Frontline healthcare workers.

5. Persons who lost family and friends secondary COVID-19 infection.

6. Persons who contact mental health services, such as telepsychiatry helplines and services.

7. Vulnerable groups such as the elderly, those at risk of intimate partner violence, as well as Lesbian, Gay, Bisexual and Transsexual (LGBT) and other social minorities.

8. People presenting to primary care settings with non-specific complaints.

STEP ॥

Screen for Mental Health Distress (MHD) using the General Health Questionnaire-12 ${ }^{20}$ (GHQ-12) in the most convenient language of the person receiving the screening. This can be done through telemedicine, online screening surveys, emergency care settings, primary and community healthcare settings.

1. Negative screen (GHQ score less than 4): Counsel and educate on preventing mental illness and promoting good mental health using the WHO guidelines, ${ }^{21}$ as well as reducing stigma related to COVID-19. For high-risk groups, proceed to step 3 despite negative screening.

2. Positive screen (GHQ score greater than or equal to 4): Provide list of options to person receiving the screening.

i. Explain the process following a positive screen.

ii. Explain the possibility of loss of anonymity for persons being screened through telemedicine means (breach of confidentiality) should severe mental health challenges be discovered on further screening as such cases would be scaled up for appropriate treatment.

iii. Request informed consent to proceed.

iv. Proceed to step 3 for application of quick screen toolkit* as assembled by the ECP research group.

\section{STEP III}

The quick screen toolkit consists of seven screening instruments which can be used for screening and severity rating of common mental disorders at the primary care level. A positive screen using any of the tools moves person assessing care to STEP IV. Any positive screen with a moderate or severe rating will be referred for face-to-face specialist psychiatric consultation only. The screening should take approximately 15-20 min.

1. Patient Health Questionnaire ${ }^{22}(\mathrm{PHQ}) 9$ for the screening of major depression.

2. Coronavirus Anxiety Scale ${ }^{23}$ (CAS) for the screening of COVID-19related anxiety.

3. Abbreviated PTSD checklist-civilian version ${ }^{24}$ (PCL-C) for the screening of post-traumatic stress disorder. 


\section{Box 1 Continued}

4. Alcohol Use Disorder Identification Test ${ }^{25}$ (AUDIT) for the screening of alcohol misuse.

5. Drug Abuse Screening Test ${ }^{26}$ (DAST) 10 for the screening of psychoactive substance abuse.

6. Columbia Suicide Severity Rating Scale ${ }^{27}$ (C-SSRS) for the screening of suicidality.

7. Global Assessment of Functioning ${ }^{28}$ (GAF) scale to assess the need for in-patient care.

STEP IV

Upscale the person seeking care to the appropriate sector for management depending on the severity of the quick screen toolkit. The management involves:

1. Psychological counselling by trained counsellors if available in particular settings for positive screens with mild severity rating (except mild rating on the C-SSRS where minimal referral is for psychological therapy).

2. Psychological therapy—-this could be through telepsychiatry means using video-conferencing or face-to-face consultation depending on the phase of the COVID-19 pandemic.

3. Psychiatric outpatient consultation for severity ratings greater than mild.

4. Psychiatric inpatient care for severity rating greater than moderate.

5. All persons seeking care are to be entered into a data system to enable to adequate follow-up. During each follow-up, a phone call is put across and person is screen to determine severity rating using previously used tool for which they screened positive. These are done on a weekly basis.

6. All data should be stored with the person's identification number, not with their name nor other identifiable information.

*Some content of the quick screen toolkit was adapted from The SAMHSAHRSA (Substance Abuse and Mental Health Services Administration and the Health Resources and Services Administration) Center for Integrated Health Solutions (CIHS) and all tools have not been validated to e-mental health use.

the measures put in place to mitigate the spread of infection). Thus, the at-risk groups become hidden and hard to reach. ${ }^{15}$ Following a review of the literature published during the COVID-19 outbreak, we were able to identify the at-risk groups as listed in Box $1^{16171819}$. These at-risk groups as well as members of the general population (with non-specific psychological complaints such as insomnia and somatising complaints) presenting at general outpatient clinics and community health centres during the pandemic should be screened for mental health distress using the General Health Questionnaire-12 (GHQ-12). We advise the use of flyers, posters and announcements on mainstream and social media on the availability of psychosocial services at the community and primary healthcare level to aid in the recruitment of patients for screening. These recruitment strategies should avoid the use of stigmatising languages and should be communicated in such a way to encourage patient turn-up. Community health workers should build rapport with potential patients in the community to encourage their use of available mental health services at health centres. These service users are further encouraged to spread the word on the availability of psychosocial services in their locality.
Benefits of toolkit

Evaluation of this toolkit can provide evidence for research approaches to community health programmes, to discover what works and why, as well as barriers and outcomes. The use of this toolkit cuts down on the time required for mental health care to reach large populations at times of pandemic as well as those in hard-toreach areas, for example, rural areas. Data collected from use of this toolkit could serve as evidence for the mental health impact of pandemics on different populations and high-risk individuals, allowing for development of mental health strategies in future pandemic preparedness.

Author affiliations

${ }^{1}$ Department of Neuropsychiatry, University of Port Harcourt Teaching Hospital, Choba, Rivers, Nigeria

${ }^{2}$ Department of Clinical Neurosciences/DIMSC, School of Medicine, Polytechnic University of Marche, Ancona, Marche, Italy

${ }^{3}$ Mental Health Center Prizren, Hospital and University Clinical Service of Kosovo,

Prizren, Kosovo

${ }^{4}$ Department of Psychiatry, American University of Beirut, Beirut, Lebanon ${ }^{5}$ CERSAME, School of Medicine and Health Sciences, Universidad del RosarioClinica Nuestra Senora de la Paz, Bogota, Colombia

${ }^{6}$ Psychiatry Department D, Razi Hospital, faculty of Medicine of Tunis, Tunis El Manar University, Tunis, Tunisia

${ }^{7}$ Addictions Service (SerD), Department of Territorial Assistance, ASL Teramo, Teramo, Italy

${ }^{8}$ Helwan Mental Health Hospital, Cairo, Egypt

${ }^{9}$ Department of Child and Adolescent Psychiatry, NYU Grossman School of Medicine, New York, New York, USA

${ }^{10}$ Unit for Social and Community Psychiatry (WHO Collaborating Centre for Mental Health Services Development), Queen Mary University of London, London, UK

${ }^{11}$ Institute of Biomedical Sciences Abel Salazar, University of Porto, Porto, Portugal

${ }^{12}$ Hospital de Magalhaes Lemos, Porto, Portugal

${ }^{13}$ Department of Psychiatry, BKL Walawalkar Rural Medical College, Sawarde,

Ratnagiri, India

${ }^{14}$ Department of Childhood and Adolescent Psychiatry, Universidade Federal do Rio Grande do Sul, Porto Alegre, RS, Brazil

${ }^{15}$ Mental Health Research Center, Department of Psychiatry, School of Medicine, Iran University of Medical Sciences, Tehran, Iran (the Islamic Republic of)

${ }^{16}$ Fidmag Research Foundation, Hermanas Hospitalarias, Barcelona, Spain

${ }^{17}$ Department of Psychiatry, Tarakan General Hospital, Jakarta, Indonesia

${ }^{18}$ Early Psychosis Intervention Programme, Institute of Mental Health, Singapore

${ }^{19}$ Department of Social and Community Health, School of Populatin Health,

University of Auckland, Auckland, New Zealand

Correction notice The article has been corrected since it was published. The author name, Rodrigo Ramalho has been updated.

Twitter Frances Adiukwu @franadiukwu

Acknowledgements The authors would like to acknowledge the Early Career Psychiatrist section of the WPA.

Contributors FNA developed the concept of this manuscript and discussed it with all coauthors (LO, DGB, SEH, JG-D, AL, PG, MN, VP-S, MPdC, RR, ALST, MS, JS-V,

ZS, GKK, RDR). FNA wrote the initial draft and all coauthors edited and approved the final version for submission. FNA is responsible for the overall content of this paper.

Funding The authors have not declared a specific grant for this research from any funding agency in the public, commercial or not-for-profit sectors.

Competing interests None declared.

Patient consent for publication Not required.

Provenance and peer review Not commissioned; externally peer reviewed.

Open access This is an open access article distributed in accordance with the Creative Commons Attribution Non Commercial (CC BY-NC 4.0) license, which permits others to distribute, remix, adapt, build upon this work non-commercially, and license their derivative works on different terms, provided the original work is 
properly cited, appropriate credit is given, any changes made indicated, and the use is non-commercial. See: http://creativecommons.org/licenses/by-nc/4.0/.

ORCID iDs

Frances Adiukwu http://orcid.org/0000-0001-5543-1746

Mariana Pinto da Costa http://orcid.org/0000-0002-5966-5723

\section{REFERENCES}

1 Douglas PK, Douglas DB, Harrigan DC, et al. Preparing for pandemic influenza and its aftermath: mental health issues considered. Int $J$ Emerg Ment Health 2009;11:137-44.

2 Pan American Health Organization. Protecting mental health during epidemics.

3 Mohammed A, Sheikh TL, Poggensee G, et al. Mental health in emergency response: lessons from Ebola. Lancet Psychiatry 2015;2:955-7.

4 Mohammed A, Sheikh TL, Gidado S, et al. An evaluation of psychological distress and social support of survivors and contacts of Ebola virus disease infection and their relatives in Lagos, Nigeria: a cross sectional study-2014. BMC Public Health 2015;15:824.

5 Ransing R, Adiukwu F, Pereira-Sanchez V, et al. Mental health interventions during the COVID-19 pandemic: a conceptual framework by early career psychiatrists. Asian J Psychiatr 2020;51. doi:10.1016/j.ajp.2020.102085

6 Ramalho R, Adiukwu F, Gashi Bytyçi D, et al. Telepsychiatry and healthcare access inequities during the COVID-19 pandemic. Asian J Psychiatr 2020;53:102234.

7 Obilade TT. Ebola virus disease stigmatization; the role of societal attributes. Int Arch Med 2015;8:1755-7682.

8 Pereira-Sanchez V, Adiukwu F, El Hayek S, et al. COVID-19 effect on mental health: patients and workforce. Lancet Psychiatry 2020;7:e29-30

9 Mental health and COVID-19 [Internet]. Available: https://www.who. int/teams/mental-health-and-substance-use/covid-19 [Accessed 14 Jun 2020].

10 World Health Organization. WHO WHO mental health gap action programme (mhGAP). WHO, 2014.

11 Auerbach J, Miller BF. COVID-19 exposes the cracks in our already fragile mental health system. Am J Public Health 2020:e1-2.

12 Haggarty JM, Ryan KD, Jarva J. Mental health collaborative care: a synopsis of the rural and isolated toolkit, 2010.

13 Ransing R, Deshpande SN, Shete SR, et al. Assessing antenatal depression in primary care with the PHQ-2 and PHQ-9: can it be carried out by auxiliary nurse midwife (ANM)? Asian $\mathrm{J}$ Psychiatr 2020;53:102109.

14 Duarte PC, Hill AE, Morley PS. Epidemiology of equine infectious disease. In: Equine infectious diseases. 2nd edn. Elsevier Inc, 2013: 515-29.

15 Ellard-Gray A, Jeffrey NK, Choubak M, et al. Finding the hidden participant. Int J Qual Methods 2015;14:160940691562142.

16 Pappa S, Ntella V, Giannakas T, et al. Prevalence of depression, anxiety, and insomnia among healthcare workers during the COVID-19 pandemic: a systematic review and meta-analysis. Brain Behav Immun 2020;88:901-7.

17 Usher K, Durkin J, Bhullar N. The COVID-19 pandemic and mental health impacts. Int J Ment Health Nurs 2020.

18 Tan BYQ, Chew NWS, Lee GKH, et al. Psychological impact of the COVID-19 pandemic on health care workers in Singapore. Ann Intern Med 2020. doi:10.7326/M20-1083

19 Galea S, Merchant RM, Lurie N. The mental health consequences of COVID-19 and physical distancing: the need for prevention and early intervention. vol. 180, JAMA internal medicine. American Medical Association 2020:817-8.

20 Goldberg DP, Gater R, Sartorius N, et al. The validity of two versions of the GHQ in the who study of mental illness in general health care. Psychol Med 1997;27:191-7.

21 Mental health and psychosocial considerations during the COVID-19 outbreak [Internet], 2020. Available: https://www.who.int/docs/ default-source/coronaviruse/mental-health-considerations.pdf [Accessed 30 Apr 2020].

22 Kroenke K, Spitzer RL, Williams JB. The PHQ-9: validity of a brief depression severity measure. J Gen Intern Med 2001;16:606-13.

23 Lee SA. Coronavirus anxiety scale: a brief mental health screener for COVID-19 related anxiety. Death Stud 2020;44:393-401.

24 Lang AJ, Stein MB. An abbreviated PTSD checklist for use as a screening instrument in primary care. Behav Res Ther 2005:43:585-94.

25 AUDIT: the Alcohol Use Disorders Identification Test: guidelines for use in primary health care [Internet]. Available: https://www.who.int/ publications-detail/audit-the-alcohol-use-disorders-identificationtest-guidelines-for-use-in-primary-health-care [Accessed $29 \mathrm{Apr}$ 2020].

26 NIDA CTN Common Data Elements [Internet]. Instrument: Drug Abuse Screening Test (DAST-10). Available: https://cde.drugabuse. gov/instrument/e9053390-ee9c-9140-e040-bb89ad433d69 [Accessed 30 Apr 2020].

27 About the Protocol The Columbia Lighthouse Project [Internet]. Available: https://cssrs.columbia.edu/the-columbia-scale-c-ssrs/ about-the-scale/ [Accessed 29 Apr 2020].

28 Endicott J, Spitzer RL, Fleiss JL, et al. The Global Assessment Scale: a procedure for measuring overall severity of psychiatric disturbance. Arch Gen Psychiatry 1976;33:766-71.

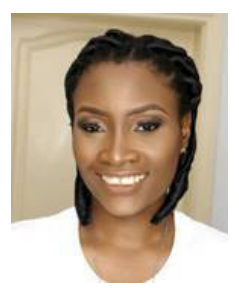

Dr Frances Adiukwu is a psychiatrist practicing in the Department of Neuropsychiatry at the University of Port Harcourt Teaching Hospital in Rivers state, Nigeria, a fellow of the West African College of Physicians (faculty of psychiatry), and a member of the National Post Graduate Medical College of Nigeria. She is also a member of the Early Career Psychiatrist (ECP) Section of the World Psychiatrist Association (WPA) and has been a recipient of the Early Career Fellowship award of the WPA in 2017. She is currently part of a COVID-19 research team under the ECP section of WPA, researching the mental health implications of the current pandemic. Her main research interests include biomarker panels for mood disorders and genetics of severe mental illnesses. 